\title{
Stochastic Sequestration Promotes Specificity in Decision Making in Single Cells
}

Ciriyam Jayaprakash ${ }^{* 1}$ and Jayajit Das ${ }^{* 2,3}$

${ }^{1}$ Department of Physics, The Ohio State University, Columbus, OH 43210

${ }^{2}$ Battelle Center for Mathematical Medicine, the Research Institute at the Nationwide Children's Hospital, Columbus, OH 43205

${ }^{3}$ Department of Pediatrics, The Wexner College of Medicine, The Ohio State University, Columbus, $\mathrm{OH} 43210$
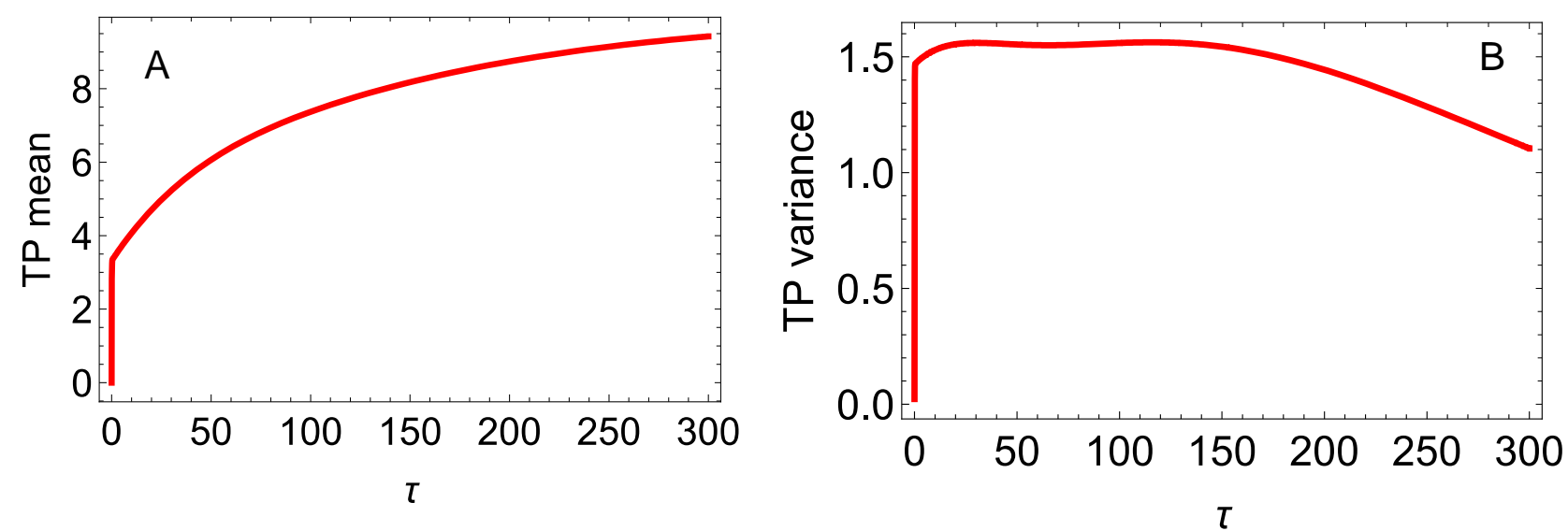

Figure S1 Variation of the (A) mean and the (B) variance of the complex TP with time where the mean and the variance are calculated from the solution of the Master Eq. (2) for the parameter values used for the case shown in red in Figure 2 in the main text. 


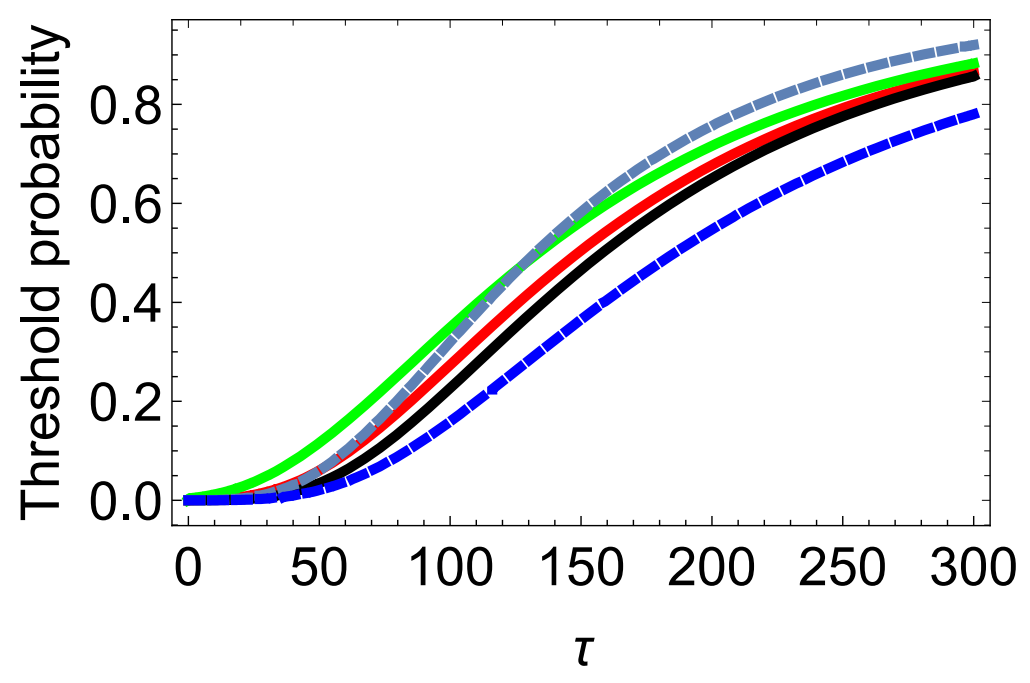

Figure S2. Sensitivity of the $\operatorname{THP}(\tau)$ vs $\tau$ variation shown in Figure 2. The parameter variations for the $\operatorname{THP}(\tau)$ vs $\tau$ data shown in red in Figure 2 in the main text are shown. The red curve in Fig. 2 is shown here in red as the reference. For the rest of the cases shown here the parameters noted here were varied, the rest of the parameters were the same as in the case shown in red in Figure 2. The following variations were carried out where $\mathrm{k}_{\mathrm{f}}$ and $\mathrm{k}_{\mathrm{r}}$ were changed by $\sim-50 \%$ and $\sim 33 \%$ and the number of decoys by $\sim \pm 10 \%$. (1) $\mathrm{k}_{\mathrm{f}}=\mathrm{k}_{\mathrm{r}}=0.45$ (green), (2) $\mathrm{k}_{\mathrm{f}}=\mathrm{k}_{\mathrm{r}}=0.20$ (black), (3) $\mathrm{N}^{0}{ }_{\mathrm{D}}=540$ (dashed dark blue), (4) $\mathrm{N}^{0}{ }_{\mathrm{D}}=660$ (blue dashed).

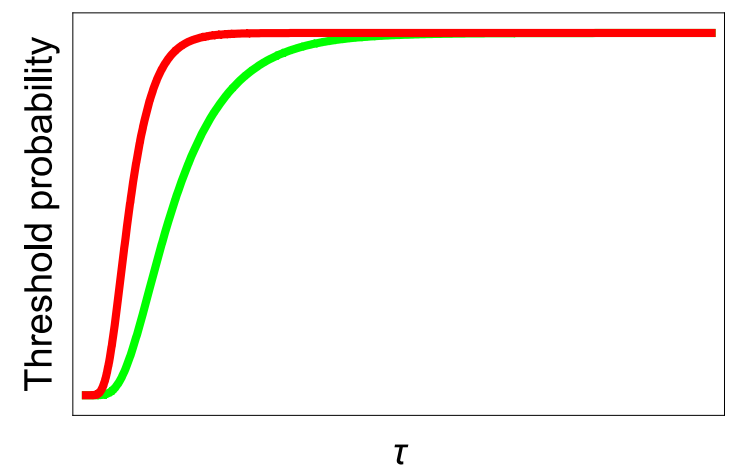

Figure S3. S-shaped variation of $\operatorname{THP}(\tau)$ with $\tau$ for finely tuned parameter values. When $\mathrm{N}_{\mathrm{P}}^{0}=10, \mathrm{~N}_{\mathrm{D}}^{0}=0, \mathrm{~N}_{\mathrm{T}}^{0}=11, \mathrm{k}_{\mathrm{f}}^{\prime}=1 / 100 \mathrm{~s}^{-1}$ and $\mathrm{k}_{\mathrm{r}}^{\prime}=1 / 300 \mathrm{~s}^{-1}$, THP $(\tau)$ shows an $\mathrm{S}-$ shaped increase with $\tau$ (green). When $\mathrm{N}_{\mathrm{T}}{ }^{0}$ is increased to 15 keeping the rest of the parameters the same the threshold decreases to a much smaller $\tau$ (red). $\mathrm{N}_{\mathrm{th}}=9$ for both the cases. 